\title{
Chorea precipitated by phototherapy as initial presentation of systemic lupus erythematosus
}

\begin{abstract}
Dear Editor,
Chorea is a well-recognised albeit rare neuropsychiatric manifestation of systemic lupus erythematosus (SLE). We describe here a case of a 67-year-old woman presenting with chorea, which affected her left lower limb and both upper limbs after phototherapy.
\end{abstract}

Chorea is an extrapyramidal movement disorder characterised by non-repetitive, abrupt, involuntary jerky movements that may be unilateral or generalised. The movements are non-patterned with variable speed, timing and direction, flowing from one body part to another and giving, in less severe cases, an appearance of fidgetiness. The unpredictable nature of chorea distinguishes it from tremor and dystonia. ${ }^{1}$ Chorea has numerous aetiologies, including structural, pharmacologic, autoimmune, metabolic and genetic. SLE is one such cause and the systemic autoimmune disorder is characterised by the presence of autoantibodies and multiorgan involvement. Neuropsychiatric symptoms are recognised manifestations of SLE and are included in classifications by the American College of Rheumatology (ACR), European League against Rheumatism (EULAR-ACR 2019) and Systemic Lupus International Collaborating Clinics (SLICC 2012). Chorea is the most common movement disorder in patients with SLE, with prevalence around $1-3 \% .^{2-4}$ While phototherapy has been reported to precipitate SLE,, 5 chorea as first presentation of SLE consequent to phototherapy has not been reported in medical literature.

We discuss the case of a 67-year-old woman with a background of quiescent rheumatoid arthritis presenting with choreoathetoid movements. The symptoms started 2 weeks after initiation of phototherapy for presumptive asteatotic eczema over her chest. She underwent a total of 5 sessions of narrow-band ultraviolet B (UVB) phototherapy, from $700 \mathrm{~mJ}$ to $1,000 \mathrm{~mJ}$ over 4 weeks. She did not have phototherapy prior to this episode. The movements initially involved her left lower limb, then progressed to both upper limbs, affecting the left more than the right. She also developed a photosensitive rash. She had no oral ulcers, joint pains or constitutional symptoms. There was no weakness or numbness, seizures, psychosis nor cognitive impairment. She had no previous history of diabetes mellitus or thyroid illness. She had no family history of movement disorders, dementia or autoimmune conditions. Examination reviewed erythema of the face sparing the eyes (where the protective goggles were worn during phototherapy). There were involuntary, variable, abrupt and rapid movements of her left upper and both lower limbs. Limb power and sensation were both preserved. Examination of the cranial nerves and gait was unremarkable. There were no cortical or cerebellar signs.

Her blood work showed anaemia (haemoglobin $10.0 \mathrm{~g} / \mathrm{dL}$ ) and leukopaenia (white blood cells $3.66 \times 10^{9} / \mathrm{L}$ ) with no evidence of haemolysis. Vitamin B12 levels were slightly low at $139 \mathrm{pmol} / \mathrm{L}$. Haemoglobin A1c and glucose levels were normal. C-reactive protein was high at $104 \mathrm{mg} / \mathrm{L}$, compared to previous level $(3.4 \mathrm{mg} / \mathrm{L})$ done during her rheumatoid follow-up. Antinuclear antibody was homogeneous with a titre of $>1 / 640$. Anti-double-stranded DNA antibody was raised at $137 \mathrm{IU}$ ( $>30 \mathrm{IU}$ considered positive), while $\mathrm{C} 3$ and $\mathrm{C} 4$ levels were low at $0.33 \mathrm{~g} / \mathrm{L}$ (normal: $0.90-1.80 \mathrm{~g} / \mathrm{L}$ ) and $0.02 \mathrm{~g} / \mathrm{L}(0.10-0.40 \mathrm{~g} / \mathrm{L})$, respectively. Anti-Ro and Anti-La antibodies were raised at $4.8 \mathrm{U}$ and $1.1 \mathrm{U}$, respectively. Schirmer's test was negative. While chorea in SLE is strongly associated with antiphospholipid antibodies (aPL), ${ }^{7}$ she tested negative for lupus anticoagulant (LAC), anticardiolipin (aCL) and anti-B2 glycoprotein antibodies. Her previous autoimmune workup and complement level were normal before current admission.

Brain magnetic resonance imaging (MRI) showed scattered fluid attenuated inversion recovery (FLAIR) hyperintensities in the bilateral periventricular subcortical white matter. Magnetic resonance angiogram did not show any evidence of vasculitis nor any abnormalities of intracranial vasculature (Fig. 1). Lumbar puncture showed normal biochemistry (white blood cells $2 / \mathrm{mL}$, red blood cell $0 / \mathrm{mL}$, protein $0.31 \mathrm{~g} / \mathrm{L}$, glucose $2.5 \mathrm{mmol} / \mathrm{L}$ ). Cerebrospinal fluid cultures, autoimmune panel and anti-B2 glycoprotein were negative. Malignancy screen (including mammography, computed tomography scan of thorax, abdomen and pelvis and serum paraneoplastic panel) was unyielding. EEG showed no epileptiform activity. A skin biopsy of her chest rash was positive for dermo-epidermal junction immunoreactivity with $\operatorname{IgM}$ and $\mathrm{C} 3$, consistent with lupus erythematosus. 


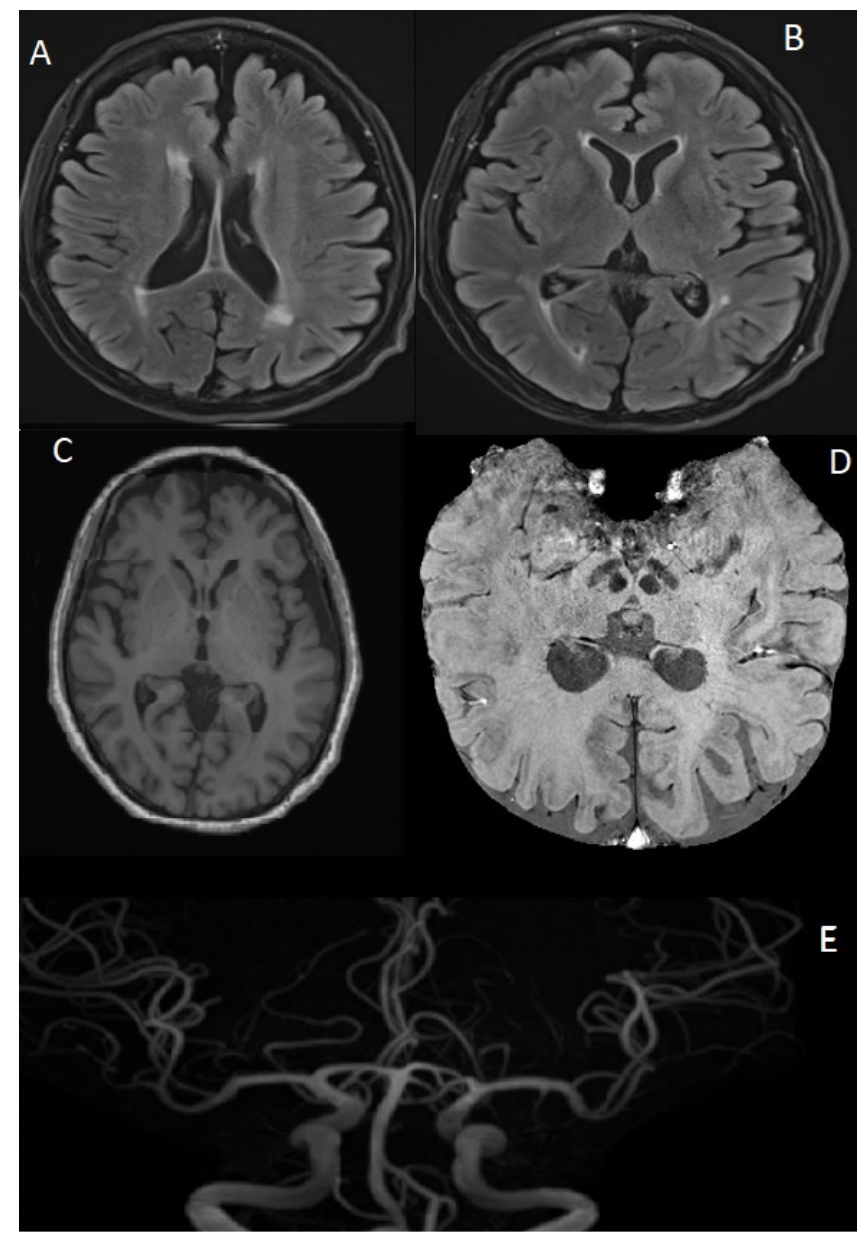

Fig. 1. The magnetic resonance brain images show periventricular white matter hyperintensities in the fluid attenuated inversion recovery setting (A and B), which is a common finding of neuropsychiatric systemic lupus erythematosus. The bilateral caudate nuclei and basal ganglia are within normal limits on T1-weighted image (C) and the nigrosome-1 is preserved on both sides on the susceptibility map-weighted image (D). The magnetic resonance angiogram shows no evidence of beading suggestive of vasculitis (E).

Tetrabenazine and olanzapine were started for symptomatic relief with some improvement in chorea. She responded well to hydroxychloroquine, methylprednisolone and cyclophosphamide with complete resolution of chorea after 2 days.

Her case is a novel description of SLE first presenting with phototherapy-precipitated chorea. Past reports have described phototherapy precipitating SLE manifestations involving the skin and joints as the presenting symptoms.

Ultraviolet radiation (UVR) is known to trigger cutaneous lupus erythematosus (CLE), with photosensitivity comprising one of the ACR diagnostic criteria for SLE. ${ }^{8}$ While the mechanisms of CLE continue to be elucidated, it is believed to be the manifestation of similar pathologic mechanisms involved in systemic disease with autoantibodies and immune complexes causing tissue damage. UVR promotes development of cutaneous lesions by augmenting apoptotic cells by lymphocytic recruitment and antibody-mediated cytotoxicity. ${ }^{9}$

While cutaneous eruptions secondary to photosensitivity after exposure to ultraviolet radiation, in particular UVB (medium wavelength, 280 to $315 \mathrm{~nm}$ ) is well recognised in literature, the relationship between cutaneous UVB exposure and systemic SLE flares are only anecdotal and suggested in case reports. Fruchter et al. ${ }^{5}$ described the clinical presentation of inflammatory polyarthropathy and urticarial vasculitis associated with haematological abnormalities as first presentation of SLE, following ultraviolet radiation in an artificial tanning device manufactured in recent years to produce higher levels of UVB. Pirner et al. ${ }^{6}$ also reported in earlier years the development of photosensitive rash and arthritis associated with haematological derangements consistent with the diagnosis of SLE in a patient who received psoralen and ultraviolet A therapy for psoriasis. Our case further supports the possible pathogenic role of ultraviolet exposure in systemic SLE flares.

Among the neurological manifestations of SLE, chorea is rare. Although chorea usually occurs during the course of SLE, it may also be the presenting feature of the illness, sometimes preceding other symptoms by several years. Chorea in SLE has been strongly associated with antiphospholipid antibodies, namely LAC, aCL and anti-B2 glycoprotein 1 antibodies. Given the low prevalence of chorea at $2 \%$ in SLE (1.3\% in antiphospholipid syndrome), ${ }^{7}$ a high index of suspicion is required to enable early diagnosis and prompt treatment to prevent further symptoms and complications related to the autoimmune process. Neuroimaging can provide further insight and assist with diagnosis of neuropsychiatric lupus. One of the most common MRI findings of neuropsychiatric SLE is periventricular white matter hyperintensities in T2 and FLAIR images, ${ }^{10}$ as in our case (Fig. 1).

\section{REFERENCES}

1. Cardoso F, Seppi K, Mair J, et al. Seminar on choreas. Lancet Neurol 2006;5:589-602.

2. Joseph FG, Lammie GA, Scolding NJ. CNS lupus: a study of 41 patients. Neurology 2007;69:644-54. 
3. Spinosa MJ, Bandeira M, Liberalesso PB, et al. Clinical, laboratory and neuroimage findings in juvenile systemic lupus erythematosus presenting involvement of the nervous system. Arq Neuropsiquiatr 2007;65:433-9.

4. Cervera R, Asherson RA, Font J, et al. Chorea in the antiphospholipid syndrome. Clinical, radiologic, and immunologic characteristics of 50 patients from our clinics and the recent literature. Medicine (Baltimore) 1996;76:203-12.

5. Fruchter O, Edoute Y. First presentation of systemic lupus erythematosus following ultraviolet radiation exposure in an artificial tanning device. Rheumatology (Oxford) 2005;44:558-9.

6. Pirner K, Rubbert A, Salinger R, et al. Bedeutung des UV-Lichtes in der Pathogenese des SLE: Kasuistik und Diskussion der Literatur [Significance of ultraviolet light in the pathogenesis of systemic lupus erythematosus: case report and discussion of the literature]. Z Rheumatol 1992;51:20-4.

7. Ricarte IF, Dutra LA, Abrantes FF, et al. Neurologic manifestations of antiphospholipid syndrome. Lupus 2018;27:1404-14.

8. Kim A, Chong BF. Photosensitivity in Cutaneous Lupus Erythematosus. Photodermatol Photoimmunol Photomed 2013;29:4-11.
9. Kuhn A, Herrmann M, Kleber S, et al. Accumulation of apoptotic cells in the epidermis of patients with cutaneous lupus erythematosus after ultraviolet irradiation. Arthritis Rheum 2006;54:939-50.

10. Provenzale JM, Barboriak DP, Allen NB, et al. Patients with antiphospholipid antibodies: CT and MR findings of the brain. AJR Am J Roentgenol 1996;167:1573-8.

Sumit Kumar Sonu ${ }^{1}{ }_{M R C P}(U K)$, Justin $\underline{\mathrm{Ng} \mathrm{CH}}{ }^{2}{ }_{M B B S}$, Si Min Chua ${ }^{2} M B B S$, M Prakash Kumar $^{3}{ }_{M R C P(U K)}$, Deidre De Silva ${ }^{3}{ }_{M R C P}(U K)$

${ }^{1}$ Department of Neurology, Singapore General Hospital, Singapore

${ }^{2}$ Department of Internal Medicine, Singapore General Hospital, Singapore

${ }^{3}$ Department of Neurology, National Neuroscience Institute, Singapore

Correspondence: Dr Sumit Kumar Sonu, Department of Neurology, Singapore General Hospital, Outram Road, Singapore 169608.

Email: sonu.sumit.kumar@singhealth.com.sg 